\title{
La fibromialgia puede desencadenarse a consecuencia de un traumatismo.
}

\author{
Fibromyalgia may develop as the result of a traumatic \\ injury.
}

\section{A. Ortega Pérez}

\section{RESUMEN}

La fibromialgia es una enfermedad conocida, con una prevalencia cercana al $2,5 \%$ de la población española mayor de 20 años, pero lo que queremos subrayar de ella es un aspecto de interés para los médicos forenses: que un porcentaje apreciable de los fibromiálgicos declara que su enfermedad les apareció poco después de sufrir un traumatismo más o menos intenso. Los enfermos con fibromialgia postraumática mostrarán las mismas manifestaciones que los que padecen fibromialgia primaria y su pronóstico será similar o algo peor que el de estos últimos. Entre los factores que determinan que un facultativo acepte el diagnóstico de fibromialgia postraumática se encuentra la especialidad de este: los reumatólogos parecen ser los más dispuestos a aceptarlo y los ortopedas, los que menos. Por otro lado, en los distintos estudios no se ha encontrado que la posible reclamación legal de estos enfermos influya en sus manifestaciones ni en su desenlace clínico. En conclusión, los médicos forenses deberían tener en cuenta la posibilidad de que el lesionado que no cura siguiendo las pautas comunes esté sufriendo una fibromialgia postraumática.

Palabras clave: Accidente de tráfico, etiología, fibromialgia, traumatismo, valoración.

\section{ABSTRACT}

Fibromyalgia is a known disease, with a prevalence of around $2.5 \%$ of the Spanish population over 20 years old. A significant aspect of the disease is that a certain amount of fibromyalgics declare that their illness appeared soon after suffering a mild or severe trauma. Patients with post-traumatic fibromyalgia show the same signs and symptoms as those with primary fibromyalgia and their prognosis is similar or somewhat worse than the latter. Among other factors determining the agreement with the diagnosis of post-traumatic fibromyalgia, is the specialty of the doctor: rheumatologists seem the most prone to accept it while the majority of orthopedists tend to reject it. According to different publications, the claim for compensation does not modify signs and symptoms or the outcome of these patients. In conclusion, forensic doctors should keep in mind the possibility that the injured patient that does not heal following the usual course of time may suffer from post-traumatic fibromyalgia.

Key words: Etiology, evaluation, fibromyalgia, traffic accident, trauma.

Correspondencia: Dr. Arturo Ortega. Área de Medicina Legal y Forense. Facultad de Medicina y Ciencias de la Salud. C/ Sant Llorenç, 21. 43201 - Reus (Tarragona). Correo electrónico: arturo.ortega@urv.net

1 Doctor en Medicina. Profesor de Medicina Legal y Toxicología. Facultad de Medicina y Ciencias de la Salud. Universidad "Rovira i Virgili". 


\section{INTRODUCCIÓN:}

Como introducción de este artículo y a la vez como síntesis de la situación que se desarrollará después, comenzaremos resumiendo lo ocurrido a una mujer a quien la fibromialgia se le desarrolló después de una lesión en el lugar de trabajo, con la particularidad de que para ella las cuestiones de indemnización y de incapacidad laboral fueron irrelevantes desde el primer momento [I].

Una mujer de 37 años, previamente sana, comenzó a sufrir dolor en la espalda y en la ingle después de levantar una caja pesada. Durante los meses siguientes, el dolor y la alodinia se diseminaron gradualmente por todo su cuerpo y empezó a sufrir cefaleas, trastornos del sueño, parestesias y alteraciones digestivas. Su dolor era constante e intenso, afectando a todas las áreas de la actividad diaria e interfiriendo con ellas.

La fibromialgia es una enfermedad conocida y estudiada desde hace cien años, aunque el término "fibromialgia" se haya acuñado hace sólo treinta. Su prevalencia entre los españoles mayores de 20 años está entre el I,5 y el 3,2 \% [2]. Aunque tanto los especialistas como los médicos de familia diagnostican la fibromialgia cada vez más a menudo [3,4,5], algunos médicos continúan dudando sobre el origen orgánico de la enfermedad [6],

Sin embargo, aquí se quiere discutir un aspecto de la enfermedad relevante para los médicos forenses, el que un porcentaje apreciable de los fibromiálgicos declara que su problema empezó poco después de sufrir un traumatismo más o menos intenso. Si la realidad de la fibromialgia aún hay quien la discute, la fibromialgia postraumática (FPT) es de asunción aún más difícil [7,8], en parte porque suele asociarse a la reclamación de indemnizaciones. No obstante, la existencia de la FPT no es una apreciación reciente, porque hace ya más de quince años Romano separó a estos enfermos en tres grupos, según sufrieran fibromialgia primaria (idiopática), asociada a otra enfermedad o secundaria a un traumatismo [9].

En esta revisión corta se expondrán los datos existentes sobre la frecuencia de la fibromialgia postraumática, cuál es el pronóstico de este subgrupo de enfermos, cotejándolo con el de todos los fibromiálgicos, qué factores influyen en la aceptación de este diagnóstico por parte de los facultativos y el efecto de la reclamación judicial en la existencia y la evolución de la enfermedad.

\section{RESULTADOS Y DISCUSIÓN:}

\section{a. Epidemiología de la fibromialgia postraumática.}

Hoy por hoy es imposible dar el porcentaje, siquiera aproximado, de fibromiálgicos que pueden indicar un traumatismo como inicio de su enfermedad, ya que la mayoría de los artículos publican series de casos de FPT y estudian o bien la influencia de su situación legal o bien su pronóstico -como los 176 enfermos atendidos entre 1980 y 1990 [ I 0] o los 46 explorados en un estudio de cohortes [ I l]-. En cualquier caso, está claro que el gran número de ejemplos publicados en la bibliografía obliga a los médicos forenses a tener en cuenta a la fibromialgia postraumática como entidad.

Greenfield et al. publicaron [1 2] que el $23 \%$ de los fibromiálgicos que habían atendido podían identificar un suceso desencadenante de su enfermedad, por lo que les diagnosticaron fibromialgia reactiva. Los autores revisaron las historias clínicas de los 127 enfermos que habían acudido a su centro en un período de cuatro años y que cumplían los criterios diagnósticos de fibromialgia; en el grupo identificaron a 29 en quienes podía señalarse un hecho concreto poco antes del inicio de sus manifestaciones, como un traumatismo, una intervención quirúrgica o una enfermedad médica.

Borenstein [13] investigó a 125 enfermos consecutivos, enviados al reumatólogo de una Unidad de Columna a lo largo de cuatro meses. A seis de ellos les diagnosticó fibromialgia primaria y a nueve, secundaria. 
Waylonis y Perkins [10] entrevistaron a fondo a 67 personas afectadas de fibromialgia postraumática. El 61 \% de los enfermos habían notado el inicio de sus manifestaciones después de un accidente de tráfico; el $13 \%$, después de una lesión en el trabajo; el $7 \%$, tras una intervención quirúrgica; el $5 \%$, después de una lesión deportiva y el $14 \%$ lo notaron después de otros traumatismos distintos.

Al-Allaf et al. [ 14] compararon a I 36 enfermos de fibromialgia y a I 52 controles, pacientes de un centro sanitario no reumatológico. El $39 \%$ de los fibromiálgicos refirieron haber padecido un traumatismo físico importante en los seis meses previos al inicio de su enfermedad, algo que había ocurrido al $24 \%$ de los pacientes control, un porcentaje significativamente menor. Los autores no hallaron diferencias significativas entre los enfermos fibromiálgicos con antecedentes de traumatismo y los que no los tenían con respecto a las variables edad, sexo, duración de la enfermedad, empleo o tarea manual.

Para terminar este apartado, un dato más: parece que la FPT ha de aparecer en los primeros meses posteriores al traumatismo, porque los que han sufrido un traumatismo en el cuello, si no desarrollan fibromialgia en el primer año, tienen pocas probabilidades de sufrirla más adelante. En la investigación de Neuman et al. [15] sólo le ocurrió a una de las 58 personas que habían padecido una lesión en el cuello tres años antes, una incidencia de fibromialgia similar a la que se da en la población general.

\section{b. Pronóstico de estos enfermos dentro del grupo de fibromialgia.}

La fibromialgia, desde un punto de vista global, es una enfermedad crónica, con unas manifestaciones que persisten al menos durante los diez años siguientes al diagnóstico [16]: dolor de moderado a grave, alteraciones del sueño de moderadas a intensas y una fatiga entre moderada y extrema. Es preciso destacar que, a pesar de que sus manifestaciones continuaban, dos tercios de los 29 enfermos estudiados por Kennedy y Felson referían encontrarse mejor o mucho mejor que en el momento del diagnóstico. La fibromialgia necesita un tratamiento continuado (el $79 \%$ de los enfermos continuaba tomando fármacos para aliviar los síntomas), aunque no sea imprescindible una supervisión médica frecuente (sólo el 52 \% habían visitado a un médico por causa de la fibromialgia en el año previo).

La investigación de Waylonis y Perkins [10] daba un resultado similar: el $85 \%$ de los fibromiálgicos continuaba sufriendo manifestaciones claras e intensas de la enfermedad varios años después del diagnóstico.

Los enfermos que padecen una FPT muestran unas manifestaciones parejas o algo más graves que las sufridas por el grupo general de fibromiálgicos. Así, los 29 enfermos que sufrían fibromialgia reactiva [12] estaban más incapacitados que los afectados por fibromialgia primaria: el $70 \%$ de aquellos habían perdido su empleo, el 34 \% habían necesitado una paga por incapacidad laboral y el $45 \%$ habían reducido su actividad física.

Turk et al. [ I I] compararon a 46 enfermos afectados por FPT con otros 46 con fibromialgia primaria. Las alteraciones físicas halladas eran comparables en ambos grupos, aunque las intensidades del dolor, de la incapacidad, de la interferencia con la vida cotidiana, de la alteración afectiva y de la reducción de la actividad física en los enfermos con FPT estaban estadísticamente relacionadas con su petición de indemnizaciones. A la vez, un número mayor de los enfermos con FPT recibían opiáceos o habían necesitado un bloqueo nervioso, fisioterapia o estimulación eléctrica nerviosa transcutánea. 
Después de doce meses de tratamiento con terapia conservadora para su dolor de espalda, los nueve enfermos que Borenstein diagnosticó de fibromialgia secundaria respondieron peor que los seis diagnosticados de fibromialgia primaria [13].

En cambio, en la investigación de Waylonis y Perkins [ [0] los 67 enfermos de fibromialgia postraumática padecían unas manifestaciones similares a las de la fibromialgia espontánea.

\section{c. Los factores determinantes del diagnóstico de fibromialgia postraumática.}

White et al. [ I7] investigaron este aspecto enviando un caso clínico a distintos profesionales sanitarios canadienses: médicos generales, ortopedas, fisioterapeutas y reumatólogos y preguntándoles si estaban de acuerdo en que a la persona se le podía haber desencadenado una fibromialgia postraumática. Las características del profesional sanitario influían en la aceptación del diagnóstico, como por ejemplo el plazo transcurrido desde la titulación (los licenciados más recientemente estaban más dispuestos a aceptarlo) o la especialidad del facultativo (el 80 \% de los reumatólogos lo aceptaba, pero sólo lo hacía el 30 \% de los ortopedas).

De todos modos, los cinco factores del cuestionario que predecían con más fuerza el acuerdo o el desacuerdo con el diagnóstico de fibromialgia postraumática fueron el número de casos de fibromialgia diagnosticados cada semana por ese profesional, el sexo del lesionado, la gravedad de las lesiones iniciales, la fuerza del impacto y los antecedentes psiquiátricos del lesionado (estos dos últimos mantenían una relación negativa con el diagnóstico).

Por otro lado, como ocurre en la mayoría de las enfermedades crónicas [1 8], algunos facultativos dan mucha influencia a los factores psíquicos en la fibromialgia. Ford [6] representa el punto de vista extremo, al defender que esta enfermedad y la sensibilidad a muchos compuestos, entre otras, eran "diagnósticos de moda", que ocultaban somatizaciones o, directamente, histeria. En su artículo describió las características de esas "enfermedades de moda": (i) quejas subjetivas y vagas, que afectan a varios aparatos y sistemas del organismo, (ii) ausencia de datos analíticos que las confirmen, (iii) explicaciones cuasicientificas, (iv) solapamiento entre varios "diagnósticos de moda", (v) manifestaciones consistentes con depresión, ansiedad o ambas, (vi) negación por el enfermo de la existencia de tensión psicosocial o atribución de esta a la enfermedad.

Es posible que algunos enfermos se acerquen a su criterio, pero pueden aportarse muchas razones a favor de la existencia real de la fibromialgia:

- La gran cantidad de casos similares en distintos países del mundo.

- El resultado de la exploración física de los puntos gatillo, algo difícil de conocer y de ser transmitido espontáneamente entre los enfermos.

- Existen otras enfermedades que afectan a varios aparatos y sistemas del organismo y están plenamente reconocidas, como ocurre con la intoxicación crónica por disolventes orgánicos.

- Las limitaciones en la explicación médica del problema o la falta de pruebas complementarias que refuercen el diagnóstico expresan nuestro desconocimiento de la enfermedad y no necesariamente que esta no exista. El artículo de Cook et al. [19] es un avance interesante con respecto a ambas cuestiones.

- La presencia de ansiedad, de depresión o de ambas manifestaciones a la vez es un efecto bastante común de la mayoría de las enfermedades crónicas.

- El enfermo puede ser plenamente sincero al explicar que no padecía una tensión psicosocial particular antes del inicio de la fibromialgia o bien que la tensión que padece en la actualidad es más una consecuencia que una causa de esta. En muchos de los casos, los afectados carecen de antecedentes psiquiátricos y las edades y las situaciones en las que se desencadenan estas enfermedades no son en absoluto las típicas de la histeria. 
Y por último, todas esas consideraciones sobre la causa de estas "enfermedades de moda" es posible que no se hicieran si la mayoría de los afectados fueran hombres, en lugar de mujeres.

\section{d. ¿Cómo influye la reclamación judicial en la enfermedad?}

Algunos autores sostienen que la epidemia actual de fibromialgia ha sido potenciada por el sistema de indemnizaciones, excesivamente generoso, de los países occidentales. No obstante, como ocurría con las manifestaciones crónicas del latigazo cervical [18], no hay datos que demuestren que la incidencia o la prevalencia de fibromialgia estén aumentando; es más, los datos existentes refutan que la indemnización y la prevalencia de fibromialgia estén asociadas [20].

Romano escribía que los enfermos que cumplen los criterios diagnósticos de fibromialgia no están simulando [9] después de estudiar las historias de 14 enfermos. A las once mujeres y a los tres hombres se les había desencadenado la enfermedad inmediatamente después de un traumatismo; de los afectados, tres fueron a juicio y once llegaron a un acuerdo extrajudicial, pero después de la indemnización el 77 \% volvieron a las consultas de un reumatólogo para continuar con el tratamiento.

Moldofsky et al. concluyeron que la situación en la que estaba la reclamación legal de los enfermos no influía en sus manifestaciones ni en su desenlace clínico [2 I]. Estudiaron a 24 enfermos con dolor crónico secundario a un accidente y apreciaron que 23 de ellos cumplían los criterios de fibromialgia. Al comparar las características de quienes tenían los problemas de indemnización judicial resueltos con las de aquellos que tenían la indemnización pendiente de resolución descubrieron que los del primer grupo eran más viejos, que sus síntomas habían durado más tiempo y que tenían mayores limitaciones laborales que los del segundo. En cambio, eran similares el resto de datos demográficos, la naturaleza del accidente, las manifestaciones, los hallazgos polisomnográficos y la incapacidad.

Para Aaron et al. [22], la percepción de haber sufrido un traumatismo físico era un determinante mayor de la percepción de una indemnización por incapacidad de lo que lo eran la percepción del trauma emocional, la gravedad de las manifestaciones o la incapacidad funcional. Pero no parece extraño que la persona enferma que pueda solicitar una indemnización lo haga, así como que los que han sufrido un trauma emocional busquen ayuda médica con mayor frecuencia $[22,23]$.

\section{CONCLUSIÓN Y APLICACIONES MEDICOFORENSES:}

Unas líneas para sintetizar los resultados de la revisión bibliográica, para extraer de ellas las conclusiones que pueden aplicarse a la práctica forense:

- Aunque no se conoce el porcentaje de fibromiálgicos que pueden indicar un traumatismo como inicio de su enfermedad, hay suficientes pruebas de la existencia de la fibromialgia postraumática.

- La FPT ha de aparecer en los primeros meses posteriores al traumatismo.

- Los enfermos con FPT padecen manifestaciones similares o algo más graves que las del grupo general.

- No todos los especialistas clínicos aceptan de igual manera el diagnóstico de FPT.

- Los factores psíquicos influyen en la fibromialgia, como ocurre en la mayoría de las enfermedades crónicas, pero la relación seguramente va en ambos sentidos.

- Los datos existentes refutan que la reclamación y la prevalencia de fibromialgia estén asociadas, al igual que ocurría con las manifestaciones crónicas del latigazo cervical. 
La conclusión más simple y más aplicable es que cuando los médicos forenses entrevisten y exploren a un lesionado que no cura o que no mejora siguiendo los plazos habituales, sino que más bien empeora, se queja de dolores progresivamente más dispersos, de sueño poco reparador, de fatigabilidad exagerada y de dolores intensos después del ejercicio o después de golpes que deberían ser leves, deben tener en cuenta la posibilidad de que al enfermo se le haya desencadenado una fibromialgia postraumática.

\section{BIBLIOGRAFÍA:}

I. Wolfe F. Post-traumatic fibromyalgia: a case report narrated by the patient. Arthritis Care Res 1994 Sep;7:161-5.

2. Carmona L, Ballina J, Gabriel R, Laffon A; EPISER Study Group. The burden of musculoskeletal diseases in the general population of Spain: results from a national survey. Ann Rheum Dis 2001;60:1040-5.

3. Soriano E, Gelado MJ, Girona MR. Fibromialgia: un diagnóstico cenicienta. Atención primaria 2000; 26:415-8.

4. Collado A, Alijotas J, Benito P, Alegre C, Romera M, Sañudo I, Martin R, Peri JM, Cots JM. Documento de consenso sobre el diagnóstico y el tratamiento de la fibromialgia en Cataluña. Med Clin (Barc) 2002;118:745-9.

5. Rivera J, Gonzalez T. The Fibromyalgia Impact Questionnaire: a validated Spanish version to assess the health status in women with fibromyalgia. Clin Exp Rheumatol 2004;22:554-60.

6. Ford CV. Somatization and fashionable diagnoses: illness as a way of life. Scand J Work Environ Health 1997;23 Suppl 3:7-16.

7. White KP, Carette S, Harth M, Teasell RW. Trauma and fibromyalgia: is there an association and what does it mean? Semin Arthritis Rheum 2000; 9:200-16.

8. Buskila D, Neumann L. Musculoskeletal injury as a trigger for fibromyalgia/posttraumatic fibromyalgia. Curr Rheumatol Rep 2000;2:104-8.

9. Romano TJ. Clinical experiences with post-traumatic fibromyalgia syndrome. W V Med J 1990;86:198-202.

10. Waylonis GW, Perkins RH. Post-traumatic fibromyalgia. A longterm follow-up. Am J Phys Med Rehabil 1994;73:403-I2.

II. Turk DC, Okifuji A, Starz TW, Sinclair JD. Effects of type of symptom onset on psychological distress and disability in fibromyalgia syndrome patients. Pain 1996;68:423-30.

12. Greenfield S, Fitzcharles MA, Esdaile JM. Reactive fibromyalgia syndrome. Arthritis Rheum 1992 Jun;35(6):678-81.

13. Borenstein D. Prevalence and treatment outcome of primary and secondary fibromyalgia in patients with spinal pain. Spine 1995;20:796-800.
14. Al-Allaf AW, Dunbar KL, Hallum NS, Nosratzadeh B, Templeton $K D$, Pullar T. A case-control study examining the role of physical trauma in the onset of fibromyalgia syndrome. Rheumatology (0xford) 2002;41:450-3.

15. Neumann L, Zeldets V, Bolotin A, Buskila D. Outcome of posttraumatic fibromyalgia: a 3-year follow-up of 78 cases of cervical spine injuries. Semin Arthritis Rheum 2003;32:320-5.

16. Kennedy M, Felson DT. A prospective long-term study of fibromyalgia syndrome. Arthritis Rheum 1996;39:682-5.

17. White KP, Ostbye T, Harth M, Nielson W, Speechley M, Teasell R, Bourne R. Perspectives on posttraumatic fibromyalgia: a random survey of Canadian general practitioners, orthopedists, physiatrists, and rheumatologists. J Rheumatol 2000;27:790-6.

18. Ortega A. Revisión crítica sobre el síndrome del latigazo cervical (II): icuánto tiempo tardará en curar?. Cuadernos de Medicina Forense 2003; 34:15-27.

19. Cook DB, Lange G, Ciccone DS, Liu WC, Steffener J, Natelson BH. Functional imaging of pain in patients with primary fibromyalgia. J Rheumatol 2004:31:364-78.

20. White KP, Harth M. Classification, epidemiology, and natural history of fibromyalgia. Curr Pain Headache Rep 2001;5:320-9.

21. Moldofsky H, Wong MT, Lue FA. Litigation, sleep, symptoms and disabilities in postaccident pain (fibromyalgia). J Rheumatol 1993;20:1935-40.

22. Aaron LA, Bradley LA, Aaron GS, Triana-Alexander M, Alexander RW, Martin MY, Alberts KR. Perceived physical and emotional trauma as precipitating events in fibromyalgia. Associations with health care seeking and disability status but not pain severity. Arthritis Rheum 1997;40:453-60.

23. Plazaola-Castaño J, Ruiz Pérez I. Violencia contra la mujer en la pareja y consecuencias en la salud física y psíquica. Med Clin (Barc) 2004 Apr 3;122(I2):461-7. 